\title{
Die Biorevitalisierung der Haut mit nativer Hyaluronsäure
}

\section{Low Molecular Hyaluronic Acid Replacement Injections Result in Smoother Skin}

Appearance, Depending on Injection Technique

\section{Zusammenfassung}

Zur Injektion steht eine neue Form der Hyaluronsäure zur Verfügung, die nicht hochvernetzt, sondern in nativem Zustand vorliegt, ähnlich der Moleküle für die topische Behandlung. Native Hyaluronsäure verteilt sich homogen in allen Hautschichten durch Diffusion. Substitution nativer Hyaluronsäure ermöglicht eine erhöhte Wasserbindung, dient dem Aufbau einer extrazellulären Matrix und optimiert die Zellaktivität ähnlich einer verbesserten Matrix bei jüngeren Erwachsenen (Biorevitalisierung). Zusätzlich sind Radikalfängereigenschaften beschrieben worden. Als Folge dieser Wirkung kommt es zu einer Verbesserung der Hauttextur, Spannkraft, Elastizität und möglicherweise zu einem positiven Einfluss auf die Zellalterung. Bedeutung hat ein solcher Aufbau der Haut dort, wo der Turgor vergangen ist und sich feine Fältchen einstellen, wie an den Wangen. Unterschiedliche Injektionstechniken werden bevorzugt, je nach Lokalisation, von Quaddeltechnik bis netzförmige Aufsättigung nach Criss-CrossTechnik und dabei in den meisten Fällen 1-2 ml intrakutan appliziert. Nach einer Anfangsphase mit ca. 2 bis 4 Injektionen im Abstand von 2-4 Wochen erfolgt eine Erhaltungstherapie alle ca. 2 - 3 Monate.

\section{Abstract}

Skin ageing is caused by endogenous biological involution like the reduction and structural changes of collagen, dermatan sulphate, GAG and hyaluronic acid leading to changes in skin texture. Hyaluronic acid is know as an active agent in moisturization. In low densities it is used as an ingredient of cosmetic products in order to bind water in the stratum corneum and as a filler for dermal augmentation, using high densities and higher molecular weights. A new low molecular hyaluronic acid of 1 Million Dalton now can be injected with 32 gauge syringes in a total of $1-3 \mathrm{ml} / \mathrm{session}$ in various techniques, starting with $1-3$ injections 3 weeks apart and 1 injection every $2^{\text {nd }}$ or $3^{\text {rd }}$ months for maintainance. Improved water binding, stimulation of collagen synthesis and free radical binding are biochemically proven results of hyaluronic acid replacement. Hyaluronic acid modulates proliferation of dermal fibroblasts in culture, provides an extracellular matrix of fibrous connective tissues that allows to build a structure of interfibrillar proteoglycan bridges and prevents oxygen free-radical damage to granulation tissue, dependent on hyaluronan molecular mass. Clinical studies proved the efficacy of this procedure, resulting in smoother skin appearance with less superficial wrinkling and less trans epidermal water loss. Injection techniques should be adapted to the degree of skin ageing, starting with intracutaneous drops in fine wrinkles (around the eyes) age 35, linear or more intense injections for older skin or cross linked infiltration of higher volumes for progressively aged skin. In combinations with other esthetic procedures Hyal provides better results. 


\section{Einleitung}

Die Lebensjahre hinterlassen Spuren - an der Haut sind sie sichtbar: die straffe Haut der Jugend vergeht, es stellen sich Fältchen ein. Gegen die sichtbare Hautalterung wurden in der Dermatologie zunehmend Behandlungsmethoden entwickelt. Zu den wichtigsten Methoden zählen Botulintoxin A, die Unterspritzung mit Fillermaterialien, Chemicalpeeling und Laserbehandlung. Die häufigsten Regionen der Faltenbehandlung sind: horizontale Stirn, seitliche Augenpartien, Krähenfüße, der Lippensaum. Dort, wo sich feine Fältchen einstellen, weil der Turgor nachlässt, wie an den Wangen, Hals und Handrücken werden Pflegeprodukte, chemical peels, sowie ablative und nicht ablative Laser eingesetzt. Hyaluronsäure ist bereits als Feuchthaltemittel in kosmetischen Pflegeprodukten bekannt, sowie als Fillersubstanz zur Unterspritzung. Seit Jüngstem kann zur Turgorverbesserung auch Hyaluronsäure injiziert werden.

\section{Hyaluronsäure - Vorkommen, physiologische Funktion und Hautalterung}

Wenn die Haut altert ist histologisch eine Abflachung der Epidermis zu beobachten, eine Reduktion von Kollagen und Glukosaminoglykanen und die Abnahme des Gehaltes an Hyaluronsäure. Während bei 19- bis 47-Jährigen der Hyaluronsäuregehalt 0,33\% beträgt, ist er bei 60 -Jährigen nur noch mit $0,015 \%$ und bei 70-Jährigen mit 0,007\% nachweisbar [1].

\section{Hyaluronsäure}

ist ein Kettenmolekül aus den Doppelzuckergrundbausteinen D-Glukuronsäure + N-Acetylglucosamin mit bis zu über 10000 Grundbausteinen. Es ist bei Tier und Mensch identisch und dient strukturellen Aufgaben in der Matrix.

Hyaluronsäure kommt im Körper zu 35\% in Sehnen und Bändern der Skelettmuskulatur vor, zu 56\% in der Haut und zu 9\% an anderen Lokalisationen, hierbei bevorzugt in der Synovia, Gelenke, Nabelschnur, Blut und Lymphflüssigkeit. Das Corpus vitreum im Auge enthält 0,1 bis 0,4 mg, Synovia 3 bis $4 \mathrm{mg} / \mathrm{g}$ Trockengewicht, Hyalinknorpelgewebe etwa $1 \mathrm{mg} / \mathrm{g}$ Trockengewicht und die Haut 7 bis $8 \mathrm{~g}$, wobei die Dermis mit 0,5 gegenüber der Epidermis mit $0,1 \mathrm{mg} / \mathrm{g}$ Trockengewicht einen höheren Gehalt zeigt.

Hyaluronsäure hat einen hohen Kompressionswiderstand, hohe Viskosität und ist damit ein idealer „Schmierstoff“, z. B. in der Synovia. Als Zellgerüstbaustein dient es dem Aufbau der extrazellulären Matrix und unterstützt auch den Raum für Diffusionswege.

Hyaluronsäure führt zu:

1. hoher Wasserbindung und Aufbau der extrazellulären Matrix, dient somit der Korrektur von Hautkonturmängeln.

2. Die Hyaluronsäurekonzentration verbessert Fibroblastenproliferation und Migration und führt damit zur Neubildung von Collagen, Elastin und endogener Hyaluronsäure

3. Aufgrund des spezifischen Molekülgewichtes ist eine Schutzfunktion auf die Fibroblasten im Sinne eines Radikalfängeref-

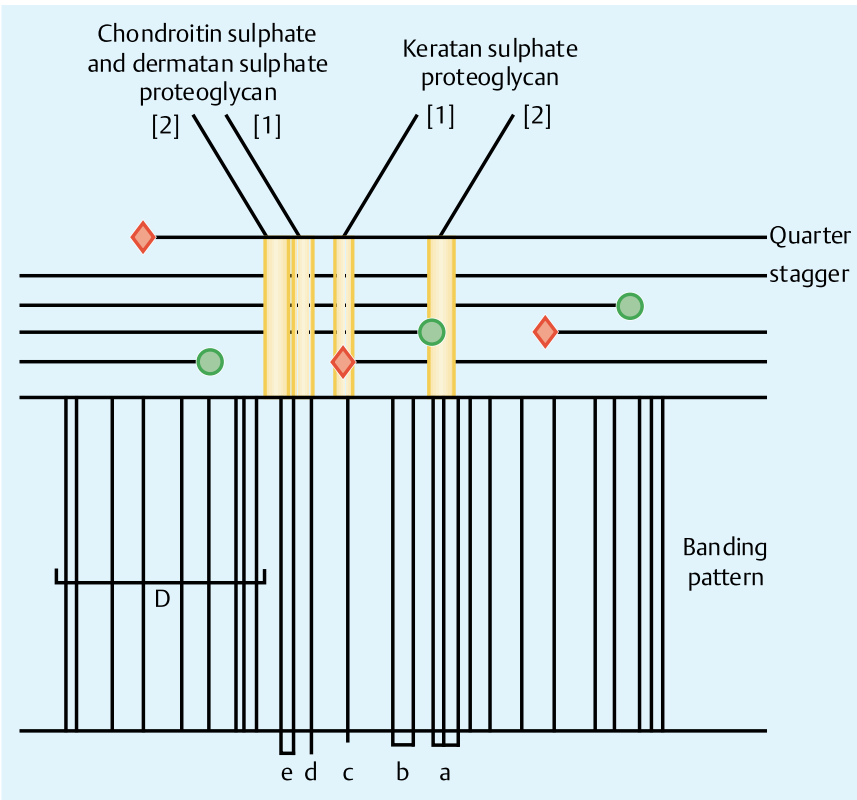

Abb. 1 Proteoglykanbrücken für die Einbindung kollagener Fibrillen in Grundsubstanz.

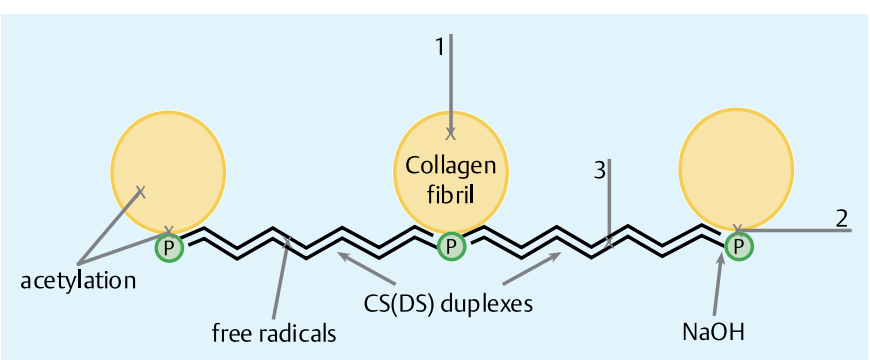

Abb.2 Einbindung kollagener Fibrillen in Grundsubstanz für die dreidimensionale Struktur der extrazellulären Matrix.

fekts nachweisbar, der einer Vorsorge der Zellalterung dienen kann.

Im Alterungsprozess nehmen nicht nur die Fibroblastenzahl und ihre Teilungsfähigkeit, sondern auch Collagen- und Hyaluronsäuregehalt ab. Folge ist eine verminderte Wasserspeicherfähigkeit mit dem Ergebnis trockener, rauher Haut, dem Einsinken des Stratum corneum, geringer Elastizität und Spannkraft. Klinisch imponieren Falten [4].

Ohne ausreichenden Gehalt an Hyaluronsäure in der Epidermis und insbesondere Dermis ist auch eine Collagenneubildung und Collageneinlagerung nicht möglich [5]. Ziel der Behandlung ist es, Hyaluronsäurespiegel im Gewebe wie in jungen Jahren zu erreichen (Abb.1).

Hyaluronsäure sorgt über kleine Proteoglykanbrücken für die Einbindung kollagener Fibrillen in Grundsubstanz und damit für die dreidimensionale Struktur der extrazellulären Matrix [6-8] (Abb. 2).

Aktiv wachsende Fibroblasten bilden Hyaluronsäure neu. Hört das Fibroblastenwachstum auf, wird auch die Neuproduktion von Hyaluronsäure eingestellt [9]. 
Hyaluronsäure selbst hat jedoch seinerseits Einfluss auf das Zellwachstum. Wird Hyaluronsäure einer zellreichen Fibroblastenkultur hinzugegeben, so lässt sich ein höheres Fibroblastenwachstum und eine höher Rate an Zellteilung erreichen als ohne Zugabe von Hyaluronsäure. Hyaluronsäure hat damit eine Aufbauwirkung. Fibroblasten bilden darunter mehr Kollagen und ermöglichen die Erneuerung der Haut.

Die Förderung des Zellwachstums ist jedoch nur bei niedrigen Hyaluronsäurekonzentrationen nachgewiesen [10], wie sie bei nativer Hyaluronsäure durch Injektionsmengen und deren Diffusion möglich ist. Werden quervernetzte Hyaluronsäuren injiziert oder große Mengen, so wirkt dies laut einer Studie von Yoneda [9] wieder bremsend auf das Zellwachstum.

Hyaluronsäure stimuliert Zellwachstum also nur in niedrigen und mittleren Molekulargewichten, wohl bedingt durch mehr Bindungsmöglichkeiten an Fibroblastenrezeptoren bei niedrigem Molekulargewicht. Hyaluronsäuren von hohem Molekulargewicht können nur in geringerer Anzahl an Fibroblastenrezeptoren binden, während die Bindung bei niedrigem Molekulargewicht für eine größere Zahl von Hyaluronsäuremolekülen möglich ist und damit ist offensichtlich eine bessere Stimulationswirkung gegeben [10]. Hierbei kommt es zu einer spezifischen Interaktion der Hyaluronsäure mit Rezeptoren der Zellmembranen. Mit CD44-Interaktion wird die Zelldifferenzierung gesteigert und mit RHAMM die Steigerung der Zellmobilität.

Darüber hinaus ist in zahlreichen Arbeiten beschrieben worden, dass Hyaluronsäure als Radikalfänger wirksam ist [11]. Es antagonisiert Superoxid-Anionen, schützt so auch den Knorpel vor Abbau und vor Lipidperoxidation und wirkt auch generell antioxidativ [12-13].

Mit zunehmender Konzentration schützt Hyaluronsäure vor einer Zellschädigung, die im Experiment durch Radikale vermittelt wurde [13].

In der Wundbehandlung zur Wundheilung [14] konnte raschere Heilung unter Zufuhr von Hyaluronsäure nachgewiesen werden. In einer Studie wurde durch freie Radikale des Phenazins eine Wundheilungsstörung künstlich erzeugt, einem Teil aber zusätzlich Hyaluronsäure lokal appliziert. Hierbei zeigte sich die Schutzwirkung gegenüber den freien Radikalen.

Hyaluronsäure wird ähnlich wie Albumin aus dem Organismus eliminiert. Nach dem Transfer via Leber, Lymphknoten und Haut wird sie mit einer Halbwertszeit von 12 bis 24 Stunden ausgeschieden [15-17].

\section{Hyaluronsäureprodukte zur Behandlung}

Hyaluronsäure wird entweder synthetisch hergestellt oder aus Hahnenkamm extrahiert und durch bakterielle Fermentation steril und allergenfrei synthetisiert.

Klinische Anwendung findet Hyaluronsäure in der Augenchirurgie, Orthopädie, Rheumatologie und plastischen Chirurgie.
Zum Volumenaufbau, z.B. an Nasolabialfalten, werden quervernetzte Hyaluronsäuren eingesetzt, die nach Injektion mehrere Monate am Ort der Applikation verbleiben, bedingt durch relative Molekülträgheit. Mit dem „Hyal-System“ steht eine neue Form der Hyaluronsäure zur Verfügung, die nicht hochvernetzt ist, sondern in nativem Zustand vorliegt. Native Hyaluronsäure diffundiert homogen in alle Hautschichten und zeigt Biointeraktivität.

Im Unterschied zu Fillern, aber auch zu den Inhaltsstoffen von Pflegeprodukten handelt es sich um ein injizierbares Material, das aufgrund seiner höheren Viskosität in die oberen Hautschichten injiziert werden kann.

Native Hyaluronsäure ermöglicht dort eine erhöhte Wasserbindung, dient dem Aufbau einer extrazellulären Matrix und optimiert die Zellaktivität, verbessert somit die Matrix des Bindegewebes. Zusätzlich sind Radikalfängereigenschaften beschrieben worden. Als Folge dieser Wirkung kommt es zu einer Verbesserung von Hauttextur, Spannkraft, Elastizität und möglicherweise zu einem protektiven Einfluss auf die Zellalterung.

\section{Niedrig molekulare Hyaluronsäure : Klinische Applikation}

Die Injektion nativer Hyaluronsäure, auch als Biorevitalisierung der Haut bezeichnet, ergänzt unsere bisher praktizierten Methoden zur Therapie der alternden Hauttextur.

Als weitere Methoden stehen uns bislang zur Verfügung:

- Pflegeprodukte mit Radikalfängereigenschaften [2],

- Chemicalpeel [3],

- Subsurfacingmethoden zur Stimulation der Kollagenneubildung ohne Ablatio der oberen Epidermis.

Mit allen diesen Systemen kann die Injektion von nativer Hyaluronsäure kombiniert werden. Während die Radikalfänger in Pflegeprodukten und Lichtschutzpräparaten der Hautalterung vorbeugen, wird durch Chemicalpeeling die epidermale Hautoberfläche geglättet und insbesondere bei jüngeren Menschen eine dermale Kollagenneubildung je nach Intensität des Peelings induziert.

Gleiche Zielsetzung verfolgt das Subsurfacing, jedoch ohne Ablation der Hautoberfläche. Im Gegensatz zum Laserresurfacing wird hier kein operativer Eingriff mehr erforderlich. Die Injektion von nativer Hyaluronsäure ermöglicht hingegen über den Weg einer erhöhten Wasserbindung und Aufbau der Matrix eine verbesserte Kollagenneubildung. Von der Matrix ist der Turgor der Haut abhängig, weniger von der Menge an Kollagen. Bisher fand sich in der ästhetischen Medizin keine Methode eine Verbesserung der Hautmatrix zu erreichen.

\section{Klinische Wirlkung nativer Hyaluronsäure}

In einer Studie [18] wurden 20 Patienten im Alter von durchschnittlich 52 Jahren zweimal im Abstand von 14 Tagen mit nativer Hyaluronsäure injiziert (Abb. 3a,b). Danach erfolgt eine Erhaltungstherapie alle 90 Tage. Hierbei kam es zu einer signifi- 

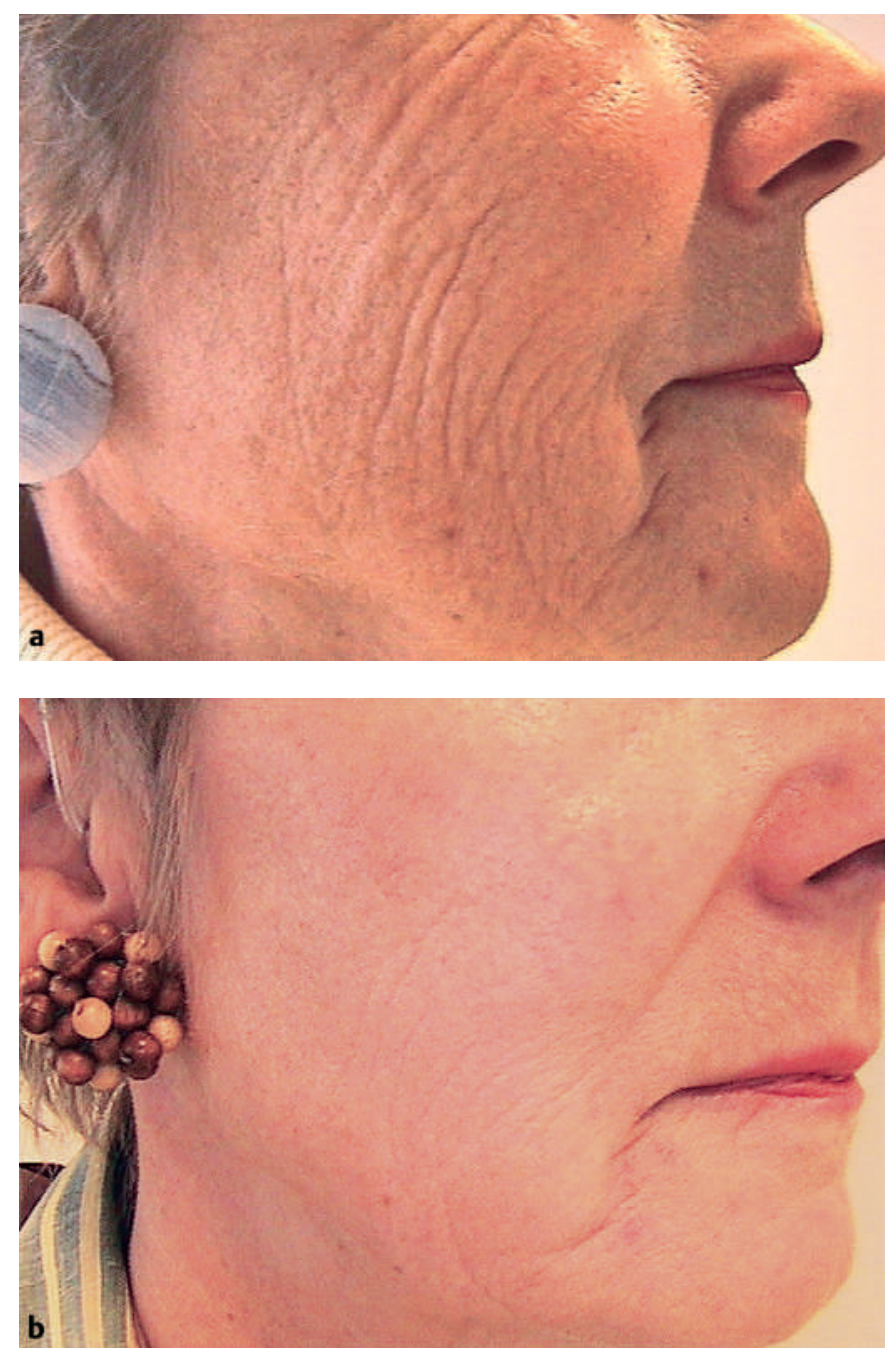

Abb.3 Alternde Haut vor (a) und nach (b) Applikation von nativer Hyaluronsäure (drei Sitzungen im Abstand von drei Wochen).

kant verbesserten Elastizität sowohl im Arzt- wie auch im Patientenurteil, bei exzellenter Verträglichkeit.

\section{Methode}

Verwendet wird eine 1,1 ml-Fertigspritze mit einer 30-g-Injektionsnadel. Empfehlenswert ist die Verwendung von $32 \mathrm{~g}$, um noch atraumatischer injizieren zu können. Die Substanz ist eine farblose hautidentische, chemisch unverändert natürliche Hyaluronsäure in Natriumsalzlösung von 1,8\% und einer Molekulargewichtsfraktion von ca. 1 Million Dalton und liegt in niedrigvisköser Lösung vor. Erstmals wurde die Substanz in Italien und Japan angewendet und ist dort seit 5 Jahren erhältlich.

\section{Praktische Durchführung}

Benötigt werden neben dem Fertigpräparat Hyal (1,1 ml native Hyaluronsäure; Herstellerfirma Fidia, Vertrieb Firma Merz), Tupfer, Kanülen der Größe 30, besser 32 g, Alkohol oder Hautdesinfektionslösung. Für die Cross-Link-Technik werden auch 27 bis $30 \mathrm{~g}$ mit 0, $4 \times 40 \mathrm{~mm}$ Länge verwendet.

\section{Injektionstechnik}

Die Injektionstechnik hängt ab von der Tiefe der Faltenbildung, dem Grad der Hautalterung und der Lokalisation. Hierbei kann man sich an der Klassifikation der Hautalterungstypen nach Glogau orientieren:

Typ der Hautalterung [19]:

- Gruppe 1 - mild (28-35 J.): keine Keratosen, feine Fältchen, wenig Make-up,

- Gruppe 2 - moderat (35-50 J.): erste aktinische Keratosen, gelbliche Hautverfärbung, frühe Faltenbildung, parallele Lachfalten, etwas mehr Make-up,

- Gruppe 3 - fortgeschritten (50-65 J.): aktinische Keratosen, gelbliche Hautverfärbung, Aknenarben, Teleangiektasien, Faltenbildung in Ruhe, immer Make-up,

- Gruppe 4 - schwer (60 - 75 J.): aktinische Keratosen + Hautkrebs, ausgeprägte tiefe Falten, Cutis laxa, gravitationsbedingte Erschlaffung, Narbenzonen, Make-up deckt nicht mehr.

Zur Verfügung steht die Quaddeltechnik streifenförmige Intrakutaninjektion im Abstand von 1 bis $1,5 \mathrm{~cm}$ und so genannte Criss-Cross-Technik, bei der intrakutan bis oberflächlich subkutan größere Mengen an Hyaluronsäure in einer netzförmigen Struktur injiziert werden.

\section{Quaddeltechnik}

Für oberflächliche Faltenbildung und Hautalterungstypen Glogau 1 bis 2 sowie insbesondere perioral und perorbital, ist die Injektion von oberflächlichen Quaddeln mit einer 32-g-Injektionskanüle am wenigsten traumatisch. Je feiner die Fältchen, um so weiter können die Abstände zwischen den Quaddeln sein (0,5 bis $2 \mathrm{~cm}$ ).

\section{Lineare Technilk}

Vor allem bei deutlich sichtbaren feinen Linien, wie die Halslinien (Abb.4), streifenförmigen Linien an den Wangen oder auch Krähenfüßen kann die native Hyaluronsäure direkt wie ein Filler unter die jeweilige Linie/Falte injiziert werden. Hierbei wird die Injektionsnadel intrakutan nach vorne geführt und beim Zurückziehen unter leichter Überkorrektur das Material appliziert. Dies kann auch in einzelnen kleineren Portionen als serielle Punktionstechnik geschehen, sei es auf dem Rückzug einer Injektion oder als einzelne etwas tiefer applizierte Quaddeln (Abb. 4).

Ganz ähnlich können nun diese Linien fächerförmig von einem zentralen Punkt aus oder auf einen zentralen Punkt hinlaufend injiziert werden, um ein Areal mit Hyaluronsäure zu sättigen. Diese Vorgehensweise bietet sich insbesondere an den seitlichen Augenpartien an oder wenn nur kleinere Wangenpartien zu behandeln sind.

Ist eine größere Menge an Material notwendig, um ein intensiveres Aufbauergebnis zu erreichen, so sollte die Criss-Cross-Technik gewählt werden. 


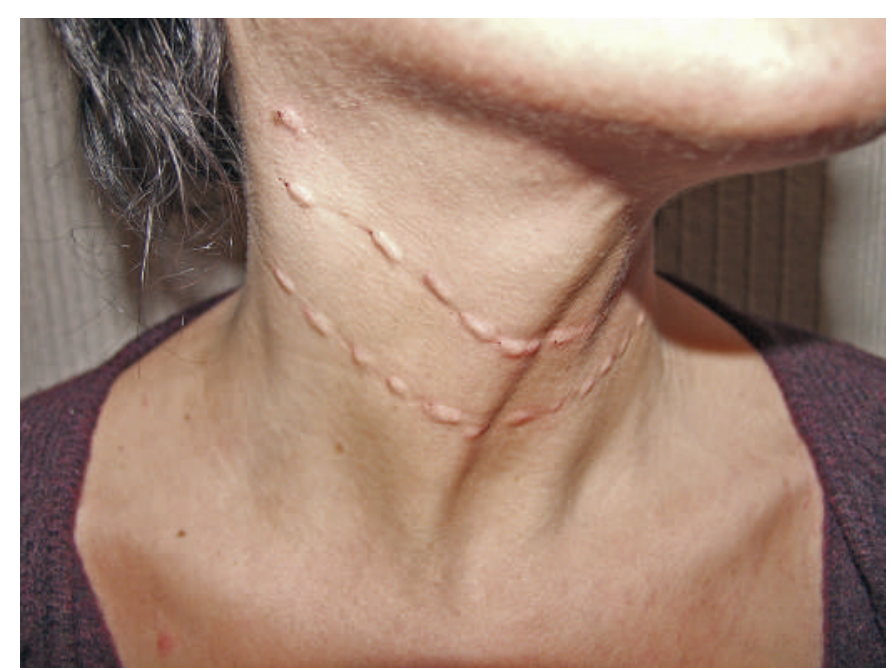

Abb. 4 Unmittelbar nach Applikation nativer Hyaluronsäure durch Quaddeltechnik in Halslinien.

Hierbei wird, wie unter lineare Technik beschrieben, zunächst im Abstand von ca. $1 \mathrm{~cm}$ streifenförmig Hyaluronsäure intrakutan appliziert, danach erfolgt mit gleicher Technik im rechten Winkel hierzu netzförmig die Injektion. Zunächst einmal horizontal, dann vertikal. Aufgrund der danach stattfindenden Diffusion ergibt sich somit eine intensive Durchtränkung.

\section{Serielle Punkttechnik}

Diese kann beispielsweise bei Faltenbildungen für einzelne Linien perioral in Frage kommen.

In Orientierung an die Hautalterungstypen dürften folgende Injektionstechniken vorherrschen:

- Glogau 1: einzelne Quaddeln mit größerem Abstand,

- Glogau 2: Quaddeln mit dichterem Abstand sowie einzelne lineare Injektionen,

- Glogau 3: lineare und auch tiefere Infiltrationen, dichte Quaddeln oder vereinzelt beginnend Criss-Cross,

- Glogau 4: Criss-Cross.

\section{Fortsetzung der Behandlung}

Zum Aufbau eines Effektes ist zunächst für geringgradige Alterserscheinungen der Haut eine in der Anfangsphase ein- bis zweimalige Wiederholung nach 2 bis 3 Wochen sinnvoll. Zum Erhalt des Ergebnisses reichen Injektionen in 2- bis 3-monatigen Abständen aus. Je nach Ausprägung und Fläche werden 2, bei geringerer Ausprägung auch nur 1 Ampulle Hyal verwendet.

Bei atonischer und deutlich gealterter Haut ist eine Anfangsphase mit ca. 3 bis 4 Sitzungen im Abstand von 2 Wochen empfehlenswert. Als Erhaltungstherapie werden dann in der Regel weitere Sitzungen zu je 2 Ampullen abhängig vom klinischen Bild nach ca. 2-3 Monaten zu empfehlen sein.

Alle Gesichtspartien mit atonischer Haut können behandelt werden. Als Besonderheit zu nennen sind die Unterlider, bei denen möglichst nur feine Quaddeln gesetzt werden, da sonst eine Hämatomneigung das sonst wenig invasive Verfahren belastet.

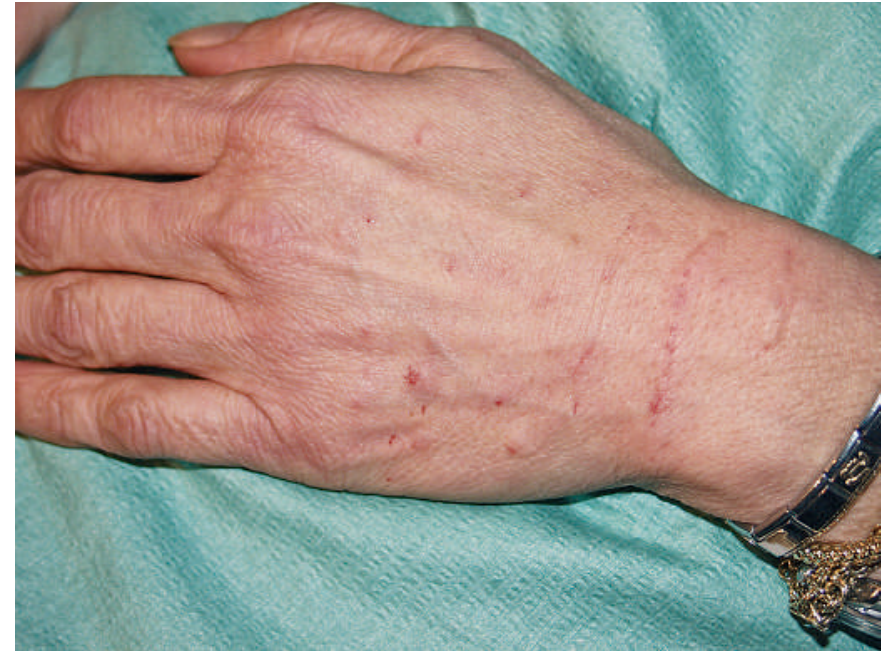

Abb.5 Applikation nativer Hyaluronsäure mit Quaddeltechnik am Handrücken.

Halslinien können durch streifenförmige Injektionen oder auch in serieller Punkttechnik geglättet werden (Abb.4). Eine Besonderheit stellen die Handrücken dar (Abb. 5). Da hier die Injektion oft etwas weniger schmerzarm ist als im Gesicht, werden in den Interdigitalräumen die Quaddeln dicht oder in streifenförmiger Injektionstechnik gesetzt. Für die Handgelenkslinien gilt das Gleiche. Um Hämatome und Schmerzhaftigkeit zu reduzieren, ist eine Kühlung vor und nach der Behandlung, ggf. die Applikation von Emla-Creme oder Betacain (USA) und die Verwendung einer sehr feinen 32-g-Kanüle empfehlenswert.

Zusammenfassend kann festgestellt werden, dass das optische Erscheinungsbild sich durch die intrakutane Applikation von nativer Hyaluronsäure (Hyalsystem) verbessern lässt. Indiziert ist die Methode bereits bei einer Haut mit ersten Alterungszeichen, ist aber auch bei fortgeschrittener Hautalterung einsetzbar. Sie erhöht Kompaktheit und Straffheit der Hautkonsistenz, verbessert den Turgor und glättet so die Hautoberfläche. Meist geben die Behandlungen dann auch ein subjektiv angenehmes Hautgefühl.

Die Verbesserung der Matrix unterstützt durch verbesserte Fibroblastenproliferation auch die Kollagenneubildung. Hyalinjektionen sind somit eine sinnvolle Kombination zu Laser-Subsurfacing, Chemicalpeeling und topischer Antiaging-Behandlung mit Produkten, die letztlich auf die Neubildung von Kollagen abzielen.

Insbesondere bei der sehr moderat und erst langfristig wirksamen Laser-Subsurfacing-Behandlung, für die zahlreiche Geräte eingesetzt werden (z.B. gepulster Farbstofflaser, Er:Glass; Er:Yag-langgepulst, Smothbeam, Nd:Yag hochfrequent [cutera], IPL, Diodenlaser), lässt sich mit Hyal ein rascher Effekt, mit Subsurfacing hingegen die Unterstützung eines langfristigen Effektes erzielen und daher auch für den Patienten subjektiv sinnvoll kombinieren.

Hyalsystem gilt auch als Einstiegstherapie für Patientinnen und Patienten, die vor invasiven Verfahren Angst haben und die eine natürliche Behandlungsform wünschen. Es stellt eine Basisthera- 
pie für die alternde Gesichtshaut dar, die in andere Verfahren integriert werden kann und bereits bei noch befriedigendem Hautturgor prophylaktisch gegen Alterserscheinungen eingesetzt werden kann.

\section{Literatur}

${ }^{1}$ Longas M. Evidence for structural changes in dermatan sulfate and hyaluronic acid with aging. Carbohydrate Research 1987; 159: 127 136

2 Keller KL, Fenske NA. Uses of vitamins A, C, and E and related compounds in dermatology: A review J Am Acad Dermatol 1998; 39: $611-625$

${ }^{3}$ Brody HJ. Chemical Peeling. St. Louis: Mosby, 1992

${ }^{4}$ Lapière $\mathrm{C}$. The aging dermis: The main cause for the appearance of „old“ skin. Br J Dermatol 1990; 122, Suppl 35: 5-11

${ }^{5}$ Doillon C, Wasserman A, Berg R, Silver F. Behavior of fibroblasts and epidermal cells cultivated on analogues of extracellular matrix. Biomaterials 1988; 9: 91 - 96

${ }^{6}$ Scott J. Proteoglycan-fibrillar collagen interactions - review article. Biochem J 1988; 255: 313-323

${ }^{7}$ Scott JE, Thomlinson AM. The structure of interfibrillar proteoglycan bridges („shape modules“) in extracellular matrix of fibrous connective tissues and their stability in various chemical environments. J Anat 1998; 193: $391-405$

${ }^{8}$ Scott E, Cummings C, Brass A, Chen Y. Secondary and tertiary structures of hyaluronan in aqueous solution, investigated by rotary shadowing-electron microscopy and computer simulation. Hyaluronan is a very efficient network-forming polymer. Biochem J 1991; 274: 699705

${ }^{9}$ Yoneda M, Yamagata M, Suzuki S, Kimata K. Hyaluronic acid modulates proliferation of mouse dermal fibroblasts in culture. J Cell Science 1988; 90: 265-273

${ }^{10}$ Smith M, Ghosh P. The synthesis of hyaluronic acid by human synovial fibroblasts is influenced by the nature of the hyaluronate in the extracellular environment. Rheumatol Int 1987; 7: 113-122

${ }^{11}$ Fukuda K, Takayama M, Ueno M, Oh M, Asada S, Kumano F, Tanaka S. Hyaluroic acid inhibits interleukin-1-induced superoxide anion in bovine chondrocytes. Birkhäuser Verlag 1997; 46: 114-117

${ }^{12}$ Artola A, Aliò J, Bellot J, Ruiz J. Lipid Peroxidation in the iris and its protection by means of viscoelastic substances (sodium hyaluronate and hydroxypropylmethylcellulose). Ophthalmic Res 1993; 25: 172-176

${ }^{13}$ Presti D, Scott J. Hyaluronan-mediated protective effect against cell damage caused by enzymatically produced hydroxyl $(\mathrm{OH}-)$ radicals is dependent on hyaluronan molecular mass. Cell Biochem Funct 1994; 12: 281

${ }^{14}$ Foschi D, Castoldi L, Radaelli E, Abelli P, Calderini G, Rastrelli A, Mariscotti C, Marazzi M, Trabucchi E. Hyaluronic acid prevents oxygen freeredical dage to granulation tissue: A study in rats. Bioscience Ediprint 1990; XXII (6): $333-339$

${ }^{15}$ Reed R, Laurent UB, Fraser JR, Laurent TC. Removal rate of [3H] hyaluronan injected subcutaneously in rabbits. Am J Physiol 1990; 259: $532-535$

${ }^{16}$ Laurent U, Lauritz B, Reed R. Catabolism of hyaluronan in rabbit skin takes place locally, in lymph nodes and liver. Experim Physiol 1991; 76: $695-703$

${ }^{17}$ Fraser J, Laurent T, Laurent U. Hyaluronan: Its nature, distribution, functions and turnover. J Intern Med 1997; 242: 27 - 33

${ }^{18}$ Di Pietro A, Di Sante G. Recovery of skin elasticity and turgor by intradermal injection of hyaluronic acid (Ial-SystemTM) by the cross-linked technique. Journal G Ital Dermatol Venereol 2001; 136: 187- 194

${ }^{19}$ Glogau RG. Chemical peel symposion. Atlanta: American Academy of Dermatology, 1990

\section{Buchbesprechung}

\section{Histamin-Intoleranz, Histamin und Seekrankheit \\ R. Jarisch (Hrsg.)}

Stuttgart: Thieme, 2. neubearb. u. erw. Aufl. 2004. 117 S., 17 Abb. Kart. 24,95€. ISBN 3-13-105382-8

Die zweite Auflage des Buches zur Histamin-Intoleranz, herausgegeben von Prof. Jarisch, Leiter eines Allergiezentrums in Wien, und mit Beiträgen von fünf Co-Autoren, wurde erweitert um das letzte Kapitel zu Histamin und Seekrankheit. Es behandelt im Hauptteil Histamin und biogene Amine, Krankheitsbilder mit Histamin-Intoleranz, Medikamentenunverträglichkeiten, z.B. bei nicht steroidalen Antiphlogistika, Kontrastmitteln und Lokalanästhetika, Histamin-Intoleranz bei Frauen mit Regelbeschwerden und in der Schwangerschaft, Neurodermitis und Histamin, die spezifische Immuntherapie, Vitamin B6, Vitamin C und Histamin. Dabei verstehen die Autoren ihre Beiträge als Ergänzung zum aktuellen Wissenstand im Hinblick auf die Rolle des Histamins, z.B. bei den Mastozytosen und nicht als Lehrbuch über Mastozytosen. Aktuelle Literatur ist bei jedem Kapitel angefügt, dennoch ist der Evidenzgrad unterschiedlich, jedoch wird dies immer objektiv dargestellt. Gut belegt sind zum Beispiel die Kapitel zur Diaminoxidase des Darmes, unserem ersten Schutzmechanismus gegen Histamin. Dieses Enzym kann mittlerweile mit kommerziell erhältlichen Kits im Blut bestimmt werden und ist bei Personen mit Symptomen auf Histamin erniedrigt. Beim Kapitel Histamin und Seekrankheit verweist der Autor darauf, dass Empfehlungspakete wie histaminfreie Diät auf See, Vitamin C und viel Schlaf in Ergänzung zum bekannten Stutgeron (Cinnarizin) noch in kontrollierten Studien zu untersuchen sind. Sehr hilfreich für die Arbeit des Allergologen sind die Tabellen zu Histamin-reichen und -armen Nahrungsmitteln, Histamingehalte in Weinen verschiedenster Reben, Champagner, Sekt und Bier. Darüber hinaus übersetzt das Buch medizinische Termini laienverständlich, so dass es auch Betroffenen empfohlen werden kann. Es wird ein „Dauerbrenner“ bleiben.

Christiane Bayerl, Wiesbaden 